\title{
Rifrattura di femore in osteoporosi severa non trattata:
} descrizione di un caso

\author{
C. Corradini, M. Ometti,J. Berneri, V. Macchi, E. Malagoli, C.Verdoia \\ Clinica Ortopedica e Traumatologica, Università degli Studi di Milano c/o I Divisione, Istituto Ortopedico G. Pini, Milano
}

D01 10.1007/s10261-010-0023-x

ABSTRACT Femoral re-fracture in untreated severe osteoporosis: a case report

In orthopaedic practice, periprothesic fractures are gaining relevance. Their incidence after first implantation is $1.5 \%$ and they increase to $6 \%$ in revisions. However, their etiology is still uncertain, as evidenced by the present case report of a femoral periprosthetic re-fracture. A female 83years-old patient, admitted to another hospital for a femoral subcapital fracture and treated with the implantation of an endoprosthesis, was discharged for a long rehabilitative period. After some time the patient had a new periprosthetic fracture, followed by prolonged bed rest due to a synthesis with long plate. Less than one year later she had her third femoral fracture of Vancouver type B1 involving the diaphysis between hip stem of the endoprosthesis and the plate implanted in the two previous occasions. During hospitalization at our institute bone turnover markers and vertebral DEXA revealed a severe poromalacic status characterized by high bone resorption and vitamin D3 deficiency related to secondary hyperparathyroidism. After surgery, in view of 3 months of wheel-chair and no weight-bearing, she started teriparatide, a bone anabolic agent, with daily calcium carbonate and vitamin D3 supplementation; 5 months after the intervention she walked on weight-bearing with crutches. At 2 years f.u. she is free from new fractures and lives and walks in autonomy with one crutch.

\section{Introduzione}

Le fratture periprotesiche stan no assumendo un'importanza sempre crescente nella pratica ortopedica. La loro incidenza è dell' $1,5 \%$ negli impianti primari, mentre riguarda il 6-8\% dei casi di revisioni [1]. Tuttavia il progressivo aumento delle fratture in protesi non è giustificabile unicamente con l'incremento annuale degli impianti [2]. Sono stati infatti individuati vari fattori di rischio sistemici e locali $[3,4]$. Peraltro i vari presidi attuati non sono risultati evidentemente utili in presenza di una rifrattura, come nel caso di seguito riportato.

\section{Caso clinico}

M.E., donna di 83 anni, affetta da vasculopatia cerebrale, portatrice di endoprotesi dell'anca sinistra con stelo anatomico cementato per una frattura sottocapitata del femore, ha appena ottenuto un discreto recupero funzionale quando riporta una nuova caduta dopo poco più di 6 mesi. Avendo riportato una frattura periprotesica distale di tipo C di Vancouver con interessamento del condilo femorale mediale, viene nuovamente ricoverata in un altro ospedale e sottoposta a riduzione e sintesi con placca condilica lunga $[1,5]$. Dopo qualche settimana in unità riabilitativa, la paziente riprende la deambulazione con l'ausilio del deambulatore. Ma, meno di un anno dopo, la paziente cade e deve essere ricoverata presso il nostro Istituto per la rifrattura diafisaria del terzo medio tipo

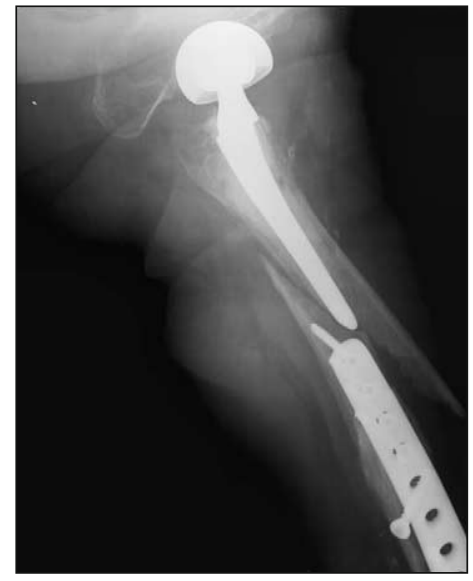

Fig. 1. Rx al momento del ricovero che evidenzia la rifrattura diafisaria del terzo medio tipo B1 di Vancouver. Si nota la rima di frattura in corrispondenza del margine libero fra la protesi e la placca

B1 di Vancouver (Fig. 1). Una volta operata per la riduzione cruenta e la sintesi con una seconda placca LCP e cerchiaggi metallici, la paziente viene sottoposta a indagini biochimiche supplementari per la valutazione del "turnover" del metabolismo osseo. Da questi esami emerge una grave osteoporomalacia, caratterizzata da elevati indici di riassorbimento, e una carenza di vitamina D con iperparatiroidismo secondario. Quindi, in considerazione della necessità di mantenere l'arto operato in scarico per 3 mesi senza incorrere in un'ulteriore riduzione della massa ossea, con l'elevazione del rischio di ulteriori fratture, si prescrive un trattamento farmacologico antiosteoporotico. Così, dalla seconda settimana post-operatoria, la paziente è stata trattata con teriparatide $20 \mu \mathrm{g} /$ die sottocute, calcio carbonato $1 \mathrm{~g} /$ die giornaliero e vitamina $D_{3} 25 \mathrm{gtt} / 1$ volta alla settimana per 18 mesi

Dopo aver effettuato fisioterapia assistita con esercizi di mobilizzazione articolare e $\mathrm{d}$ rinforzo isometrico degli arti inferiori, la paziente ha eseguito, a 45 giorni dall'intervento, un controllo radiografico con evidenza di formazione iniziale di callo osseo; mentre a 90 giorni una seconda radiografia ha evidenziato la guarigione della frattura (Fig. 2). Allora alla paziente è stata consentita la deambulazione assistita con carico progressivo, dapprima con girello e poi con l'ausilio delle stampelle fino a 5 mesi. Quindi, avendo recuperato una buona autonomia funzionale, ha ripreso la deambulazione con un bastone

La ripetizione a distanza de "marker" biochimici del "turnover" osseo ha permesso $\mathrm{d}$ rilevare la riduzione significativa degli indici di riassorbimento, l'aumento significativo degli indici di neoformazione ossea la normalizzazione dei livelli di vitamina $\mathrm{D}$ e paratormone.

Nel follow-up a oltre 2 anni la paziente non ha presentato segni di nuove fratture da fragilità, ha mantenuto i parametri del metabolismo osseo ne "range" di normalità per la fascia di età, non ha riferito nuove cadute né difficoltà funzionali maggiori di quelle con-

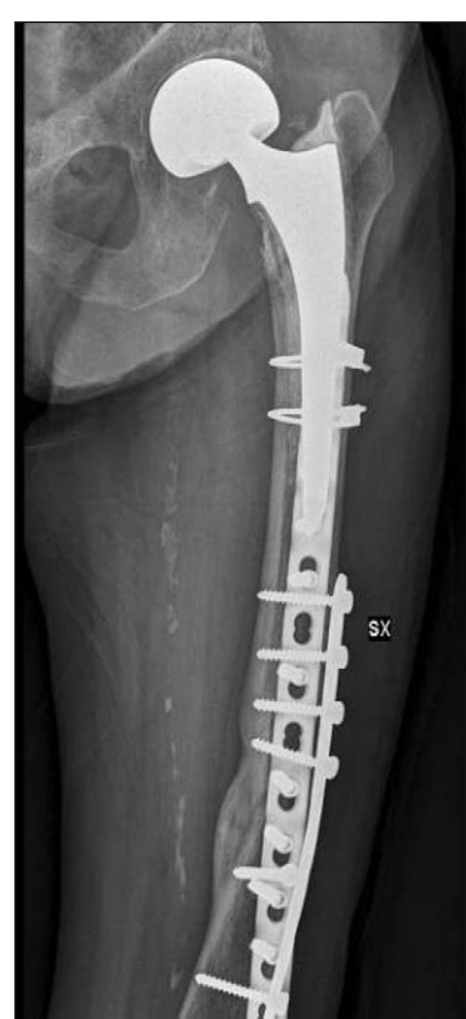

Fig. 2. Rx di controllo a distanza che dimostra guarigione della frattura con buona osteointegrazione dei mezzi di sintesi e stabilità dell'impianto protesico seguenti ai pregressi accidenti cerebrovascolari.

\section{Discussione}

Il caso appena descritto di rifrattura del femore è emblematico del fatto che in pazienti osteoporotici, fino a quando non si migliora la qualità del l'osso, il susseguirsi di eventi traumatici porta a fratture con sempre maggiore frequenza [6]

Pertanto nell'osteoporosi pro vata, data l'importanza di un primo evento al femore come fattore predittivo di nuovi eventi che possono incidere gravemente sulla qualità della vita e sui costi socioeconomici derivanti, è fondamentale impostare un adeguato trattamento farmacologico [7]. Infatti molti studi hanno dimostrato come la prescrizione di una terapia antiosteoporotica con bisfosfonati dopo il trattamento chirurgico di una frattura di femore sia stato utile per ridurre il rischio di una seconda frattura [8].

In questo caso si è deciso di utilizzare un farmaco antiosteoporotico di recente immissione in commercio, teriparatide. Esso è il metabolita attivo del paratormone che, somministrato quotidianamente per via sottocutanea, stimola direttamente la neoformazione ossea, come evidenziato dal rapido aumento dei marcatori dell'attività osteoblastica. Rispetto agli antirias sorbitivi, la cui azione quasi esclusiva è quella di ridurre il rimodellamento osseo, gli anabolici dell'osso possono aiutare la guarigione della frattura permettendo al paziente un carico più precoce e di conseguenza un recupero funzionale più rapido $[9,10]$. Il limite all'uso è dato dalla prescrizione, che deve essere fatta in strutture ospedaliere autorizzate e secondo la Nota 79 CUF-AIFA, che prevede l'associazione non temporale della frattura femorale con cedimenti vertebrali.

\section{Conclusioni}

Sulla base di tali considerazioni, nel futuro potremmo considerare la possibilità di trattare con farmaci anabolici dell'osso i soggetti con fratture periprotesiche, in modo da ridurre la probabilità di andare incontro a ulteriori fratture accelerando il processo di guarigione, consentire loro un più rapido recupe

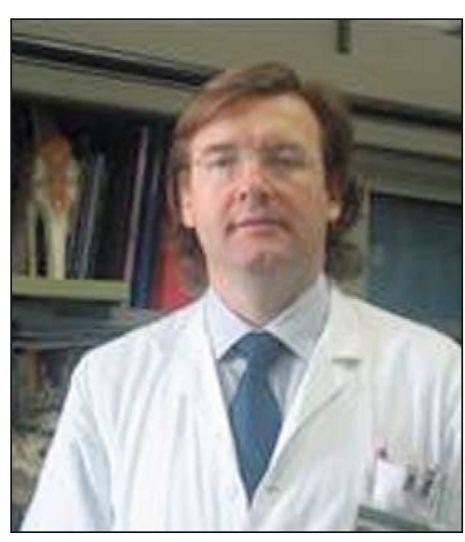

C. Corradini

ro funzionale e quindi un più veloce ritorno alla vita quotidiana.

\section{Bibliografia}

Old AB, McGrory BJ, White RR, Babikian GM (2006) Fixation of Vancouver B1 peri-prosthetic fractures by broad metal plates without the application of strut allografts. J Bone Joint Surg Br 88:1425-1429

Ricci WM, Haidukewych GJ (2009) Periprosthetic femoral fractures. Instr Course Lect 58:105-115

Beals RK, Tower SS (1996) Periprosthetic fractures of the femur. An analysis of 93 fractures. Clin Orthop 327:238-246

Kelley SS (1994) Periprosthetic femoral fractures. J Am Acad Orthop Surg 2:164-172

Chakravarthy J, Bansal R, Cooper J (2007) Locking plate osteosynthesis for Vancouver Type $B 1$ and Type $C$ periprosthetic fractures of femur: a report on 12 patients. Injury 38:725-733

Johnell O, Kaufman J, Cummings $S$ et al (2007) Recommendations for care of the osteoporotic fracture patient to reduce the risk of future fracture. World Orthopedic Osteoporosis Organization (WOOO) Gardner MJ, Flik KR, Mooar P, Lane JM (2002) Improvement in the undertreatment of osteoporosis following hip fracture. J Bone Joint Surg Am 84:13421348

Torgerson DJ, Dolan P (1998). Prescribing by general practitioners after an osteoporotic fracture. Ann Rheum Dis 57:378-379

Greenspan SL, Resnick NM, Parker RA (2005) Early changes in biochemical markers of bone turnover are associated with long-term changes in bone mineral density in elderly women on alendronate, hormone replacement therapy, or combination therapy: a three-year, 